\title{
LA HISTORIA DE LA EDUCACION Y LA PEDAGOGIA EN LA FORMACION DE DOCENTES
}

\section{Juan Carlos Torres Azócar*}

El propósito de este ensayo es presentar algunas reflexiones sobre la importancia y la necesidad de integrar la historia de la educación y la pedagogía en los programas de formación de docentes. Para ello haré referencia, en primer lugar, a ciertos aspectos que han caracterizado la constitución de esta historia sectorial como campo de investigación y, en segundo lugar, a sus posibilidades como disciplina académica, es decir, como temática específica a enseñar y como actividad heurística en la formación inicial de educadores.

\section{LA HISTORIA DE LA EDUCACIÓN Y LA PEDAGOGÍA COMO CAMPO DE INVESTIGACIÓN.}

"Y esa historia es todavía un campo por roturar; una clave de explicación apenas explorada". M. Tuñón de Lara.

En Colombia, los estudios históricos sobre la educación y la pedagogía son recientes. La mayoría de investigadores coinciden al afirmar que la especificidad de este campo de investigación empezó a configurarse en la segunda mitad de los años setenta. Desde entonces se ha transitado "el duro camino que conduce de un "tema" a verdaderos problemas de investigación", como planteó Renan Silva al referirse a las difíciles condiciones iniciales de este proceso.

La mirada histórica sobre los hechos educativos exigía delimitar un ámbito propio de indagación; identificar áreas y temas relevantes, formular los problemas y las preguntas iniciales que fueran dando sentido a ese nuevo "objeto" de estudio en construcción; desentrañar nuevas fuentes, construir archivos, afinar las teorías y los métodos; conformar y consolidar grupos de investigadores; entrar en diálogo con otras disciplinas y saberes que contribuyen a la comprensión y explicación del pasado, en particular del pasado educativo; y obtener un reconocimiento en el mundo académico y en la historiografía nacional, dos ámbitos en los cuales la investigación histórico-educativa ha sido ignorada, desconocida o sólo parcialmente tratada. De ello dan cuenta algunos investigadores al referirse a esta desigual relación : "La historia de la educación no ha ocupado un lugar privilegiado dentro del quehacer historiográfico nacional y sólo en contadas ocasiones, la reconstrucción histórica de los hechos educativos ha sido abordada con las

\footnotetext{
* Prof esor Departamento de Ciencias Sociales. Universidad Pedagógica Nacional.

${ }^{1}$ Silva, Renán: “La investigación histórica sobre educación en Colombia : Un balance provisional”. Ponencia presentada en el Tercer Seminario Nacional de Investigación en Educación. Bogotá, abril 14, 15 y 16 de 1986. Memorias. Universidad Pedagógica Nacional e ICFES, p. 120.
} 
herramientas propias de la disciplina histórica; quizás por esta razón los historiadores la han considerado hermana menor, aunque a veces en sus disquisiciones sobre la cultura alcancen a vislumbrar la importancia de reconstruir lo que la historización de la educación tiene para aportar en este ámbito".

Llama la atención el descuido que ha existido sobre este tema dado el papel estratégico y funcional que ha jugado la educación en los principales hechos y momentos de la historia colombiana: en la formación de la nacionalidad, en los procesos de modernización económica e institucional del país ; de integración territorial, de homogeneización cultural y en la constitución de mecanismos de control político, ideológico y social; y, sobre todo, en la formación de una mentalidad moderna, a través de la difusión y apropiación del pensamiento científico, mediante la circulación de las ideas, las teorías y los métodos más actualizados en distintas áreas del conocimiento. Estas razones, entre muchas otras que se pueden mencionar, parecerían ser suficientes para despertar el interés de los académicos y para motivar nuevas exploraciones del pasado que amplíen nuestro conocimiento histórico desde el anális is de las relaciones entre educación, sociedad y cultura.

De hecho fue en el ámbito educativo, a tra vés de la formación universitaria de profesores, que se formó una nueva intelectualidad académica en el país. En la Escuela Nomal Superior (1936-1951) se pretendió formar profesores que tuviesen tanto un dominio de las disciplinas que enseñaban como una sólida preparación pedagógica y didáctica y que al mismo tiempo supiesen contextualizar la enseñanza según las características socio-culturales del medio donde se realizaba la acción educativa. Además de tener una vida institucional marcada por la tolerancia ideológica que posibilitó la libertad de pensamiento y la circulación de las teorías más actualizadas en las áreas enseñadas, se creó un ambiente propicio para la generación de nuevas teorías sociales y de elementos de interpretación de la realidad nacional. La fundación de institutos anexos de investigación, pemitieron la formación de un bagaje científico significativo, especialmente en las ciencias sociales y humanas, lo cual contribuyó decisivamente para la institucionalización de esas disciplinas en el país. ${ }^{3}$

Veamos ahora algunos rasgos particulares que han perfilado la historia de la educación y la pedagogía como campo de investigación. Los antecedentes son verdaderamente precarios. De las pocas obras existentes sobre el tema, anteriores a la década del setenta, solamente merece ser destacada "La evolución educativa en Colombia", de Luis Bohórquez Casallas, no porque represente un aporte historiográfico importante sino porque desde su publicación en 1956 ha sido una obra de consulta obligada, "porque es un texto que ha

\footnotetext{
${ }^{2}$ Herrera, Martha y Low, Carlos: Los intelectuales y el despertar cultural del siglo. El caso de la Escuela Normal Superior: Una historia reciente y olvidada", Santafé de Bogotá, Universidad Pedagógica Nacional, 1994, p. 11.

${ }^{3}$ Herrera, Martha: "Historia de la formación de profesores en Colombia: Una búsqueda de identidad profesional", en Formacao de Professores. A experiencia internacional sob o olhar brasileiro. Editora Autores Associados, Nupes, Brasil, 1998, p. 265. (Texto original en portugués; traducción libre). 
circulado, no sólo en las Escuelas Nomales y Facultades de Educación, en donde por algunos años ha sido texto para la enseñanza, sino también entre públicos más amplios; e incluso, citado y utilizado sin crítica ni rubor por algunos historiadores que parecen aceptar la versión o los hechos. Y desde luego que con este texto se ha presentado de manera insistente el proceso por el cual después de afirmado un error se continúa por muchos años repitiéndolo, pero con mayor énfasis". Sin embargo, el autor de esta cita, reconoce que la obra de Bohórquez "es, dentro de la his toria más tradicionalista, la más significativa por cuanto ofreció una "visión global" del proceso, y por los múltiples usos que ha permitido (y permite)". Además, se le reconoce una relativa virtud al abordar la cotidianidad escolar "ya que la escuela y los hechos escolares han permanecido marginados de la indagación histórica". 4

Esta última aseveración nos remite a examinar las tendencias y los intereses predominantes en la historiografía colombiana en las últimas décadas. Desde aquellas posiciones más tradicionalistas centradas principalmente en los hechos políticos y jurídicos, con su culto a los héroes y a los poderosos y su desconocimiento de las mayorías y los "débiles"; pasando por una historiografía más preocupada por los grandes hechos estructurales de carácter económico y social, pero anclada en determinismos y en rígidos principios explicativos; hasta las distintas vertientes de la historia social agrupadas en la llamada Nueva Historia, con su inclinación por lo colectivo; todas estas corrientes historiográficas descuidaron no sólo la múltiple complejidad de los hechos educativos sino también la riqueza de prácticas sociales de distinto orden que desde todos los ámbitos y con múltiples manifestaciones irrumpían en el país, sin ser reconocidas por los historiadores como temas "dignos" de investigación.

Son éstas otras dimensiones de la realidad las que vienen interpelando a la disciplina histórica en todo el mundo, llevándola a extender sus dominios, a empujar sus fronteras, a descubrir o explorar nuevos territorios sin abandonar aquellos que le son más familiares, pero renovándose, abriéndose a la diversidad, a los cambios que se producen en los sentidos y en las significaciones sociales y culturales del mundo contemporáneo. Los intensos debates librados a nivel internacional y nacional, dan cuenta de los cambios ocurridos en la investigación his tórica, en la ampliación y redefinición de sus objetos de análisis, en la búsqueda de nuevas vías y perspectivas de comprensión, interpretación y explicación del pasado, en la naturaleza misma del quehacer del historiador y en su propia formación académica. ${ }^{5}$ Es el reconocimiento de la existencia de nuevos objetos,

\footnotetext{
${ }^{4}$ Silva, Renán: Op. Cit., 121 y 134. Además de la precaria historiografia tradicionalista sobre la materia, Silva cita otros trabajos que jugaron un papel significativo en la configuración de esta historia sectorial: "El ideal de lo práctico. El desafio de formar una élite empresarial en Colombia" de Frank Safford (1976/1989); "Elementos para la historia de la educación en Colombia en el siglo XX" de Ivon Lebot (1972); "Evolución de la política educativa durante el siglo XX. Primera parte, 1900-1957" de Alfredo Molano (1984); y principalmente, "La educación en Colombia 1918-1957. Una historia social, económica y política” de Aline Helg (1984/1987). Una mención aparte merecen los trabajos de Jaime Jaramillo Uribe "La historia de la educación como historia de la cultura" y "El proceso de la educación, del virreinato a la época contemporánea" (1980).

${ }^{5}$ De la amplia bibliografia sobre estos debates podemos consultar: Fontana, Josep. La historia después del fin de la historia, Barcelona, Cnítica, 1992; Tovar, Bernardo (comp.), La historia al final del milenio, Santafé de Bogotá, Universidad Nacional, 1994; Ortiz, Carlos y Tovar, Bernardo (eds.), Pensar el pasado, Santafé de Bogotá, Universidad Nacional - Archivo General de la Nación, 1997. 
nuevos discursos, nuevos sujetos, nuevas prácticas y nuevas instituciones cuya 'historización' pueda permitir una mejor comprensión del presente. Como señala Mauricio Archila: "Hoy nos interesa resaltar lo sobresaliente y al mismo tiempo lo común y corriente. Esto cuestiona la concepción tradicional del hecho histórico y de la misma disciplina", agregando que, "Hoy pensamos la sociedad más como un conjunto de múltiples voces que como una unidad homogénea ... En otras palabras, no nos es suficiente contar con la versión hegemónica; es necesario mirar otras versiones, contestatarias o no, con el fin de enriquecer más la reconstrucción de los hechos. La historia, de esta forma, se amplía en dimensiones impensables hace unos años".

La Historia de la Educación y la Pedagogía no ha sido ajena a los debates y a la renovación de la historiografía nacional, por el contrario su propia constitución como campo de investigación se ubica en esta dinámica, pero enriqueciéndola desde la búsqueda de alternativas teóricas y metodológicas para abordar el pasado, alternativas que se han ido construyendo y validando en el proceso mismo de 'historiar' el hecho educativo en las condiciones concretas de nuestra propia realidad. A respecto, resultan pertinentes dos apreciaciones que pueden ayudar a caracterizar este proceso; la sugestiva metáfora de Eric Hobsbawm, citada por Archila, para referirse al oficio de reconstruir el pasado: "El arte del historiador es armar el rom pecabezas de ese pasado, con la particularidad de que el rompecabezas puede ser amado de múltiples formas"; 7 y aquella que nos recuerda que "el oficio del historiador es una actividad del presente". Es en este sentido que se debe expresar la responsabilidad social del historiador frente a los hechos históricos y su compromiso ético-político frente a los ciudadanos y a la sociedad en que vive. "La comprensión del presente no es posible sin el conocimiento histórico y de allí se desprende nuestra legitimidad intelectual y nuestra responsabilidad ética. Somos los funcionarios de la memoria de la sociedad y manejamos las daves del pasado. Somos, en fin, constructores de la sociedad presente. En esta tarea colaboramos con los otros científicos sociales pues nuestros destinos están cruzados, así recorramos caminos diferentes".

El campo de la investigación histórico-educativa se ha construido entonces en el renovado terreno de la his toria social y cultural (indagando principalmente en el ámbito de las ideas, la formación de una intelectualidad académica, las mentalidades, los imaginarios sociales y educativos), ${ }^{10}$ y en el terreno de lo que se ha llamado "historia de los saberes", perspectiva orientada a desentrañar las

\footnotetext{
${ }^{6}$ Archila, Mauricio : "El historiador ¿o la alquimia del pasado?, en Ortiz, Carlos y Tovar, Bernardo. Pensar el pasado, pp. 75-123.

${ }^{7}$ Ibid., p. 79.

${ }^{8}$ Ibid., p. 84.

${ }^{9}$ Ibid., p. 85

${ }^{10}$ En esteterreno podemos ubicar algunos trabajos de laUniversidad Pedagógica Nacional, en especial las investigaciones de Diana Soto Arango sobre la educación durante el período colonial, y de Martha Herrera sobre el papel de los intelectuales en la creación y transmisión de la educación y la cultura. Ver, Herrera, M. y Low, C. "Los intelectuales y el despertar cultural del siglo. El caso de la Escuela Normal Superior. Una historia reciente y olvidada”, Bogotá, UPN, 1984.
} 
condiciones sociales de existencia de la pedagogía en nuestro medio, su especificidad como saber y como práctica. ${ }^{11}$

El punto de partida más evidente de este proceso lo podemos ubicar en la creación del Proyecto Interuniversitario "Hacia una Historia de la Práctica Pedagógica en Colombia", a finales de los años setenta. Consta de cuatro proyectos desarrollados respectivamente por las universidades implicadas : "La práctica pedagógica en la Colonia", Universidad Pedagógica Nacional ; "Los Jesuitas como maestros", Universidad Nacional de Colombia; "La práctica pedagógica del siglo XIX", Universidad de Antioquia ; y "La práctica pedagógica del siglo XX", Universidad del Valle. Las raíces se encuentran en el proyecto Filosofía y Pedagogía organizado por Olga Lucía Zuluaga en la Facultad de Educación de la Universidad de Antioquia (1975-1978). Las reflexiones del grupo de investigadores agrupados alrededor de es te proyecto partieron del abordaje de los principales textos y autores clásicos, y examinando las relaciones de la pedagogía con la filosofía, lo que pemitió iniciar el ordenamiento de los conceptos metodológicos y de las problematizaciones de la Pedagogía, y perfilar un planteamiento epistemológico que sin aislarse del contexto socioeconómico y político, recuperara la especificidad de lo pedagógico en la "reconstrucción histórica". El interés se centró entonces en tratar de "reconstruir" la historia de la práctica pedagógica y rescatar la historicidad de la pedagogía, es decir, el carácter positivo tanto de las fuentes como de la discursividad acerca de la enseñanza, para analizarla como saber, para analizar sus procesos de formación como disciplina y para analizar la práctica del saber pedagógico en nuestra sociedad; identificando, a su vez, al maestro como sujeto de ese saber. En síntesis, se trata de comprender "que la historiografía pedagógica no se construye por fuera de la pedagogía misma". 12

Estas reflexiones llevaron a tomar una posición crítica frente a las historiografías existentes hasta entonces sobre la educación en Colombia y a plantear un corte radical con ellas. Se criticaba el énfas is socioeconómico de estos trabajos y la preeminencia de la sociología como marco del análisis y de la definición de los objetos de análisis, subsumiendo el hecho educativo en concepciones generalizadoras de la enseñanza cuya "evolución" se establece en periodizaciones provenientes del orden jurídico, del orden político o del orden económico, "como si no tuvieran his toria propia dentro de otras historias, y como si fuesen carentes de su propia discursividad"; además, se les critica el hecho de tener como fuente única o principal el conjunto de políticas educativas, tanto estatales como religiosas, que han sido a su vez las instituciones fundamentales del quehacer educativo en el país. Para superar el énfasis estadístico en el terreno

\footnotetext{
${ }^{11}$ En este terreno se ubican autores como Olga Lucía Zuluaga y Jesús A. Echeverry de la Universidad de Antioquia; Alberto Martínez, Alejandro Alvarez, Jorge O. Castro y Carlos Noguera de la Universidad Pedagógica Nacional; Stella Restrepo de la Universidad Nacional; Humberto Quiceno y Guillermo Sánchez de la Universidad del Valle, entre otros, y una abundante producción investigativa de amplia circulación en los medios académicos.

${ }^{12}$ El corpus teórico y metodológico de esta propuesta se encuentra básicamente en el libro de Olga Lucía Zuluaga, Pedagogía e Historia, Bogotá, Ediciones Foro Nacional por Colombia, 1987. Es una referencia imprescindible para comprender esta perspectiva, así como otros trabajos posteriores de los investigadores del gnupo que aportan nuevos elementos para el análisis histórico dela educación y lapedagogía.
} 
educativo, que reforzó el desplazamiento de la pedagogía por las ciencias de la educación y su atomización entre las ciencias humanas, se planteó que había que abordarla como historia epistemológica. "Las historias de la Pedagogía están conformadas por datos biográficos, análisis de los cambios en el método de enseñanza, apartes sobre legislación educativa con referencias al momento político en el cual se emiten, y apartes de historias de las ideas con sus habituales conceptos de influencia, cris is, evolución, interés y toma de conciencia para explicar los cambios histónicos".

A través de la siguiente cita podemos comprender el sentido de esta perspectiva: "La historia de la práctica pedagógica posibilita el análisis de las formas de lo dicho, ya que en las prácticas de saber en la enseñanza, la Pedagogía produce formas de enunciación de los saberes. Esta aproximación permite la apropiación de un capítulo de nuestra historia cultural, al convertir en objeto de análisis la memoria del saber institucionalizado y de los sujetos que mediante él participan de una práctica. Tratamos entonces de indagar en su funcionamiento institucional la interioridad del discurso pedagógico y de su práctica, lo cual implica considerar la Pedagogía no en sí misma, sino en un complejo de relaciones que posee su propia historicidad y que reencontramos en su condición de saber pedagógico. Sólo un estudio de carácter histórico puede mostrar el lugar que ha ocupado la práctica pedagógica entre las prácticas de saber". ${ }^{14}$

Los aportes del grupo de historia de la práctica pedagógica han ejercido una relativa influencia en algunas Facultades de Educación, "haciendo escuela" y propiciando la formación de nuevos historiadores de la educación; y también han tenido una influencia significativa en el Movimiento Pedagógico, dotando al magisterio de un importante cuerpo teórico para analizar y enfrentar las políticas educativas, para redefinir su papel en la acción educativa, para recuperar la práctica y el saber que le es propio, para reconstruir su identidad cultural. Es to ha sido reconocido en los siguientes téminos por la organización magisterial: "La investigación sobre la educación y la pedagogía tomó nuevos rumbos; en las universidades públicas más importantes frente a la historia oficial de la educación, entendida como historia legislativa o casuística, aparecieron nuevas tendencias para dar cuenta sistemáticamente de las condiciones y las formas que ha vivido la docencia en el país. Y frente a la investigación psicologista-experimental, se empezaron a conocer otras tendencias más andadas en lo social, más ambiciosas para entender la magnitud de la tarea docente, más próximas a la tradición de saber qué sustenta la pedagogía que aquellas que sólo pretendían imponemos un rol de administradores curriculares para cumplir las metas del desarrollo señaladas exógena y verticalmente. Sus resultados y la interacción de los investigadores con el magis terio han venido aportando desde entonces nuevos puntos de vista para

\footnotetext{
${ }^{13}$ Ibid., pp. 13-17.

${ }^{14}$ Ibid., p. 27. 
una revaluación del modelo de práctica pedagógica propuesto desde la tecnología educativa". ${ }^{15}$

El relativo desarrollo alcanzado por la historia de la educación y la pedagogía en los últimos años en Colombia, se expresa en la persistencia y solidez de algunos núcleos de investigadores ${ }^{16}$, en la elaboración de una amplia gama de investigaciones realizadas des de distintos enfoques teóricos y metodológicos, en la construcción de archivos pedagógicos sistematizados, en el continuo incremento de publicaciones y en la realización de eventos especializados o en la participación en eventos que, como los Congresos de Historia, han incluido comisiones específicas sobre esta temática. Estos avances se han reforzado también con la creación de programas de maestría y de doctorado en algunas universidades, los cuales convocan a profesionales de distintas disciplinas interesados en esta materia, augurando la expansión y el fortalecimiento de la misma en los próximos años.

En el campo específico se ha podido convocar a los investigadores mediante la realización de varios eventos de carácter nacional, tales como: el I Coloquio de Historia de la Educación Colombiana (1989, Bogotá), el II Coloquio (1996, Tunja), y el III Coloquio (1998, Popayán). Gran parte de estas actividades se han realizado durante los años noventa con el auspicio y el impulso del Programa de Maestría en Historia de la Educación y la Pedagogía de la Universidad Pedagógica Nacional, y del Programa de Doctorado en Ciencias de la Educación, convenio que agrupa a las universidades de Cauca, Cartagena, Caldas, Distrital "Francisco José de Caldas", Nariño, Tecnológica de Pereira, y Pedagógica y Tecnológica de Tunja, y de la Red Colombiana de Historia de la Educación y de la Sociedad de Historia de la Educación Latinoamericana, ambas de reciente creación.

Otro aspecto destacable es la vinculación académica de los investigadores colombianos con pares internacionales. En 1992, se organizó el I Congreso Iberoamericano de Historia de la Educación Latinoamericana (Santafé de Bogotá, Colombia). Des de entonces, cada dos años se ha venido realizando este evento en diferentes países, el II Congreso (1994, Cam pinas, Brasil) donde se constituyó la Sociedad de Historia de la Educación Latinoamericana; el III Congreso (1996, Caracas, Venezuela); el IV Congreso (1998, Santiago, Chile); el V Congreso se realizará el año 2000, en España. Actualmente, la Sociedad de Historia de la Educación Latinoamericana integra 20 países latinoamericanos (en los cuales se están organizando las respectivas sociedades nacionales) y a 3 europeos. Además, se edita anualmente el Boletín de Historia de la Educación Latinoamericana, donde se destaca el creciente interés por la materia en distintos

\footnotetext{
${ }^{15}$ CEID-FECODE. “El Movimiento Pedagógico. Anotaciones para una balance”. Educación y Cultura, № 29, Santafé de Bogotá, 1993,pp.53-54.

${ }^{16}$ Me re fiero principalmente a la continuidad y a la unidad teórico-metodológica que agrupa a los investigadores del macroproyecto Historia de la Práctica Pedagógica en Colombia, pues en las otras perspectivas histórico-educativas no encontramos programas de investigación en sentido estricto, sino más bien proyectos aislados, algunos de largo alcance. Esta dispersión ha impedido un avance mayor del campo deinvestigación.
} 
medios académicos y la dinámica adquirida por este campo de investigación en el continente.

Al respecto, resulta interesante revisar las temáticas abordadas en los Congresos para establecer los intereses predominantes y el amplio espectro que se abarca: "Historia de las políticas educativas", "Historia del pensamiento pedagógico", "Historia de la institucionalización de la escuela", "Historia de la Universidades", "Historia de los saberes pedagógicos", "Historia de los modelos educativos de las instituciones eclesiásticas", "Historia de la difusión y de la enseñanza de la ciencia", "Nuevas alternativas en la enseñanza de la historia", "Historia de los movimientos docentes y estudiantiles", "Historia de los movimientos sociales y educación popular", "Transferencia cultural y educación de pueblos indígenas", "Tendencias teóricas y metodológicas en la investigación histórica de la educación", "Centros y redes de información educativa en América Latina" ; y la presentación de nuevas líneas de investigación como la de "His toria de la educación de la mujer" y la de "Inmigración, exilio y educación en América Latina".

Para cerrar esta parte del ensayo, podemos afimar que la Historia de la Educación y la Pedagogía, desde la configuración de un ámbito propio de indagación, ha contribuido a la renovación de la investigación histórica en Colombia. Ubicada en los terrenos y en los debates contemporáneos de la disciplina histórica, enriquece la comprensión de la realidad social y cultural desde la compleja dimensión de los hechos educativos en sus más diversas manifestaciones. Expresado por algunos investigadores, "debe también decirse que la historia de la práctica pedagógica, a pesar de estar entrelazada con las historias de las prácticas económicas, políticas y sociales, no es directamente reductible ni descifrable desde ellas. En especial, las historias sociales y de las mentalidades pueden enriquecerse con la consideración de las prácticas de saber como estrategias de gobiemo social y de autogobierno de sí, prácticas más complejas que la mera constricción económica, la represión política o la imposición unilateral de modelos de conducta. Ese trabajo de enriquecimiento debe comenzar por ampliar - y en cierto modo desbloquear- el rango dado a la noción misma de lo social en esas his torias". 17

Los aportes se expresan también, mediante la incorporación de nuevas fuentes, temas y problemas que no habían sido valorados por la historiografía nacional : la infancia, la niñez, la adolescencia, la familia, la escuela, la educación, la pedagogía, la enseñanza, la instrucción pública, las prácticas escolares, los maestros, los saberes, el cuerpo, la higiene, la raza, la población, la moral, las normas, la ciudad como espacio educativo y pedagógico, los medios masivos de comunicación. Cada vez se incorporan nuevos objetos de indagación, nuevos espacios y nuevos agentes educativos, y se establecen nuevas relaciones con las

\footnotetext{
${ }^{17}$ Ver Sáenz, Javier, Saldariaga, Oscar y Ospina, Armando. Mirar la infancia : pedagogía, moral y modernidad en Colombia, 1903-1946, Ediciones Foro Nacional por Colombia, Ediciones Uniandes, Editorial Universidad de Antioquia, Medellín, 1997, Vol. 2, p. 398.
} 
diversas expresiones sociales y culturales históricamente constituídas, apareciendo a la vista de todos como un campo de investigación dinámico y promisorio. Sin embargo, el camino apenas comienza y falta mucho por recorrer, aún son muchos los tópicos que esperan indagación, ampliación o clarificación; a manera de ejemplo mencionaré algunos: Ias relaciones Iglesia-Estado-Educación; las relaciones familia-escuela-comunidad, la historia de la participación de la familia en la vida escolar; la historia del tiempo y del espacio escolar, la historia de la arquitectura escolar; la historia de la infancia y de la juventud; la historia de la mujer y la educación. Aún no tenemos un trabajo de síntesis que nos muestre una perspectiva histórica de la formación de maestros. No tenemos una historia social y cultural de la profesión docente. Está por escribirse la historia de la enseñanza de las distintas disciplinas que tienen presencia en el ámbito académico, sobre todo en los programas de fomación de docentes. La historia del currículo, de las disciplinas y de los manuales escolares. La historia de la educación por niveles y modalidades. Faltan estudios monográficos y estudios exploratorios que aborden ciertos temas específicos, que abran nuevos problemas a la reflexión de la comunidad académica y que alimenten aquellos trabajos más sistemáticos, de largo aliento, tales como la his toria de las instituciones, la historia de las disciplinas y saberes, la historia educativa de grandes períodos, o las historias educativas regionales. Sobre estas últimas debemos destacar la creciente - aunque dispersa producción investigativa en el campo de la historia de la educación en distintas regiones del país.

Una perspectiva de trabajo que apenas se inicia en América Latina, es la referida a la investigación en educación comparada, particularmente en el análisis histórico y comparado de la educación en distintos períodos, regiones y países del continente, por ejemplo, en relación con la formación y evolución de los sistemas educativos; las políticas y reformas educativas; las políticas curriculares; la historia de las instituciones; la formación de maestros; las disciplinas académicas, los modelos de enseñanza y los libros de texto; las fuentes y archivos documentales, entre otros. Al

respecto, en el capítulo introductorio de un libro de reciente aparición, sus compiladores abogan por una historia comparada de la educación en América Latina para superar la ingenuidad de estudios que tienden a igualar procesos por un afán meramente unificador, y que llegan incluso a sacrificar "el necesario rigor investigativo que constata procesos históricos divergentes o contradictorios entre si". 18

Esta aventura intelectual emprendida hace dos décadas está demandando hoy su propia historia. Se necesita un trabajo de síntesis, un balance bibliográfico detallado que dé cuenta del camino, o mejor, de los caminos recorridos en el proceso de construcción y consolidación de la historia de la educación y la pedagogía como campo de investigación. Este puede ser el medio propicio para

\footnotetext{
${ }^{18}$ Martínez, Alberto y Narodowski, Mariano (comps.), Escuela, historia y poder. Miradas desde América Latina, Buenos Aires, Ediciones Novedades Educativas, 1996. Un ejemplo relevante de este tipo de investigaciones lo encontramos en: Messina, Graciela, Cómo seforman los maestros en América Latina,UNESCO, Santiago de Chile, 1996.
} 
hacer público el "acumulado de conocimiento", el estado de la reflexión teórica y metodológica, las tendencias y los enfoques predominantes, las fuentes y los archivos utilizados, la difusión y circulación de los trabajos, las perspectivas de futuro y los nuevos derroteros a seguir. Es indudable la relevancia y la utilidad que una obra de estas características tendría para investigadores y docentes, así como para enriquecer los programas de formación de educadores en el país, mediante la recuperación permanente de la memoria his tórica en este terreno.

\section{LA HISTORIA DE LA EDUCACIÓN Y LA PEDAGOGÍA COMO DISCIPLINA ACADÉMICA.}

"Es el presente el que ilumina el pasado".

G. Bachelard.

Se ha planteado acertadamente que la historia del maestro en Colombia es la historia de una paradoja. Una paradoja frente a su saber y frente a su quehacer. Esta situación expresa la contradicción entre lo que se le exige y lo que se le da, entre la fomación que recibe y las condiciones en que realiza su trabajo, entre la falta de reconocimiento social e intelectual y las posibilidades reales que pemitan su realización pers onal y profesional. Se le entrega la inmensa responsabilidad de formar hombres y conciencias pero se desconoce prácticamente la importancia social de su trabajo. Se le exige una gran capacidad profesional pero la formación que se le ofrece se restringe casi exclusivamente a la parte operativa del conocimiento. Se le exige una vocación supuestamente innata, pero no se le reconoce una identidad intelectual. ${ }^{19}$

Esta paradoja tiene que ver fundamentalmente con lo que ha sido y es su formación. Históricamente el maestro ha transitado por dos clases de instituciones : Las Escuelas Normales, donde el predominio del método ha limitado el sentido de su acción social a un saber hacer; y en las Facultades de Educación, donde el predominio de orientaciones profesionalizantes le ha asignado la función de administrar procesos curriculares e institucionales normativizados. De hecho esta doble vía de formación, establecida funcionalmente para responder a "las exigencias cambiantes del desarrollo económico y social del país", y mediatizada por los intereses y las relaciones de poder predominantes en cada época, corresponde a otro aspecto de la paradoja: la escisión de una profesión, entre maestros y licenciados.

Esta escisión -iniciada en los años treinta- se hace más evidente a partir de los años sesenta, cuando "puede decirse que factores relacionados con la multiplicación de las Facultades de Educación, -como respuesta a la expansión y especialización del sistema educativo-, así como la introducción de la Tecnología Educativa, la fragmentación de los saberes en torno a la educación y la pedagogía, y la baja calidad de muchas de las instituciones, dificultaron las

\footnotetext{
${ }^{19}$ Martínez, Alberto y Alvarez, Alejandro: "El maestro y su formación : la historia de una paradoja", Educación y Cultura, No 20, Bogotá, julio de 1990, pp. 5-8. 
posibilidades de que el maestro que se formaba en el nivel universitario, lo hiciera como un intelectual que domina los saberes específicos, los sis tematiza y proyecta a la luz de su fomación pedagógica, al tiempo que es capaz de ubicar su acción dentro de un entorno social y cultural específico ... De este modo se des dibuja su identidad profesional y el campo de su acción se amplía a titulados que provienen de distintas áreas profesionales, los cuales empiezan a dictar clases en el nivel secundario, nomalista y universitario, sin ningún requisito de formación pedagógica". 20

De esta manera, y al amparo de normatividades estatales excesivamente flexibles respecto a quienes pueden ejercer la profesión docente, distintas personas se disputan con el maestro el ámbito de la enseñanza. Si no existen criterios claros que delimiten el ejercicio de la profesión y definan su función social desde un saber y un quehacer específico alrededor del cual pueda construir su identidad, entonces surge inevitablemente la pregunta: ¿ para qué maestros ?. Esta pregunta nos lleva a centrar el análisis en las instituciones formadoras de docentes, específicamente en las Facultades de Educación, y en el tipo de formación que en ellas se imparte, porque es el ámbito donde "podemos recoger las experiencias históricamente acumuladas, apropiarnos creativamente del saber que allí se ha sedimentado, y a la luz de nuestras actuales circunstancias históricas, construir un nuevo modelo de fomación en el que se recupere, o se resignifique la identidad del maestro como intelectual, como profesional de la enseñanza y del saber pedagógico". ${ }^{21}$

La problemática ha sido diagnosticada y evaluada suficientemente en las últimas décadas, desde dis tintos ámbitos y perspectivas de análisis, arrojando una producción considerable de estudios y documentos de diversa índole que tratan de dar cuenta de la llamada "cris is de las instituciones formadoras de docentes". 22 in embargo, las verdaderas soluciones a los problemas detectados siguen postergadas, reconociendo inclusive que a pesar de la historia vivida, de la experiencia acumulada en el país en relación con la fomación de los educadores y de la ampliación de la oferta de programas, se evidencia la persistencia de problemas en diferentes ámbitos: excesiva proliferación, desarticulación y dispersión en los programas de formación, ofertas de baja calidad y competencia predominantemente mercantilista entre estas, incoherencia y vacíos en las políticas y la legislación referidas a la formación y al desarrollo social y profesional

\footnotetext{
${ }^{20}$ Herrera, Martha. "Los maestros y la formación universitaria: un siglo de tradición en Colombia”, Enfoques Pedagógicos, Vol. 2, Nº 3, Santafé de Bogotá, marzo de 1995, p. 30.

${ }^{21}$ Ibid., p. 31.

${ }^{22}$ Ver: Kurmen, A. y Briones, G. Evaluación de las escuelas normales en Colombia. Bogotá: MEN-PNUDUNESCO,1978, 3 vol; Tezanos de Araceli, Maestros: Artesanos intelectuales. Estudio crítico de su formación, Bogotá, Universidad Pedagógica Nacional, 1985; Muller de Ceballos, I. La lucha por la cultura. La formación del maestro colombiano en una perspectiva internacional, Santafé de Bogotá, 1992, 2 vol.; ICFES. La orientación pedagógica en los programas de formación de docentes. Reunión de Expertos. Memorias. Bogotá, 1988; ICFES. La orientación pedagógica en los programas de formación de docentes. Reunión de Decanos. Memorias. Bogotá, 1989; CEID-FECODE-UPN-OEI. Primer Encuentro Nacional de Instituciones Formadoras de Educadores, Memorias, Bogotá, 1991; MEN-ICFES. Reestructuración de Facultades de Educación, Bogotá, 1991; entre otros, y más recientemente, MEN. Lineamientos generales para la formación de maestros, Documento de trabajo. Santafé de Bogotá, 1994 y 1995; y Niño Díez, J. et. al. Hacia un sistema nacional de formación de educadores. Serie Documentos Especiales. MEN, Santafé de Bogotá, 1998. 
del educador, poca claridad de criterios académicos para orientar la formación de educadores, carencia de rigor pedagógico, escaso compromiso con la investigación educativa y pedagógica, mantenimiento de parámetros transmisionistas y aplicacionistas que luego el maestro reproduce en su acción docente, desarticulación de los programas con la realidad educativa del país, las políticas y tendencias educativas y con el contexto vital de los alumnos. ${ }^{23}$

Las sucesivas reformas implementadas para mejorar la situación personal, social y profesional del maestro no han brindado respuestas ni alternativas satisfactorias, persistiendo la inequidad de las condiciones de reconocimiento social y la desidia estatal e institucional para atender "la cualificación y dignificación de los educadores". Sin desconocer la responsabilidad que en este proceso le compete a los propios educadores, la persistencia de esta situación hace que la escuela y los educadores sigan siendo objeto de las más severas críticas y responsabilizados o culpados por la crisis generalizada del país. Esta es una his toria que se ha repetido recurrentemente en las últimas décadas, donde se cambian algunos elementos y razones de justificación pero se mantiene el mismo "chivo expiatorio".

En cuanto a los fundamentos que han caracterizado la formación, el documento en referencia señala críticamente varios as pectos que ameritan su consideración, en tanto la perspectiva de superación de los mismos constituye la base de las actuales deteminaciones legales y de las consideraciones teóricas establecidas para la reforma de los programas de formación de educadores: "Reduccionismo de la pedagogía a esquemas instrumentales y poca comprensión de su carácter disciplinario e interdisciplinario, inexistencia de comunidades académicas en pedagogía y de estados del arte que den razón de sus avances nacionales e internacionales, ausencia de debate sobre enfoques y modelos pedagógicos en general y de formación de educadores en particular, precariedad de estímulos y débil sistematización de la investigación acerca del maestro, desarticulación entre la investigación educativa y los currículos de formación de educadores, hegemonía del sentido profesionalizante en la formación del educador, poco compromiso con el desarrollo de las capacidades del educador para comprender integralmente al alumno y para ser capaz de articular las condiciones culturales, sociales y ambientales donde realizará su acción formadora". ${ }^{24}$

Estas críticas, que deben ser el punto de partida de la reflexión y de la acción propositiva por parte de quienes estamos comprometidos con la formación de docentes, conforman un complejo e ineludible entramado de temas y de

\footnotetext{
${ }^{23}$ Algunos de estos elementos fueron tomados del documento que relaciono a continuación por considerar que ofrece una buena síntesis de la problemática en cuestión: Consejo Nacional de Acreditación. Criterios y procedimientos para la acreditación previa de los programas académicos de pregrado y de especialización en educación, MEN. Santafé de Bogotá, Junio de 1998, pp. 7-11.

${ }^{24}$ Ibid., p. 9. Ver también el Decreto Número 272 de 1998. "Por el cual se establecen los requisitos de creación y funcionamiento de los programas académicos de pregrado y posgrado en Educación ofrecidos por las universidades y por las instituciones universitarias, se establece la nomenclatura de los títulos y se dictan otras disposiciones". 
problemas, el cual debe ser la base de un verdadero programa de investigación histórica, educativa y pedagógica para las Facultades de Educación.

La historia hace posible rastrear en el pasado los rasgos de constitución del hecho educativo, el cambio incesante y discontinuo de las instituciones, los sujetos, los discurs os y las prácticas que lo han caracterizado en cada momento, con el propósito de comprender su significado actual en la sociedad colombiana; es decir, "para dar lugar a la dilucidación crítica de las condiciones socio-his tónicas que han hecho y hacen posible lo que acontece / nos acontece". Esta preocupación por el presente, por "distinguir entre lo que ya no somos ..." y "lo que vamos y estamos siendo", plantea una perspectiva diferente de indagación del pasado, "la intención deliberada de construir otro modo de acceder teóricamente a la historicidad constitutiva de la educación como espacio particular de lo social". ${ }^{25}$

Los retos planteados nos llevan a centrar la atención en las posibilidades formativas de la Historia de la Educación y la Pedagogía, propósito final de este ensayo. Revisando algunos antecedentes podemos constatar que en Colombia ésta temática se constituyó precisamente como disciplina académica al ser incorporada a los planes de estudio de las escuelas nomales, tal como ocurrió en otros países. ${ }^{26}$ El Plan de Estudios para las Escuelas Normales, establecido por el Decreto 1972 de 1933, incluía la asignatura His toria de la Pedagogía, ubicada en el sexto y último año, con una intensidad horaria semanal de 4 horas. El Plan de Estudios de las Facultades de Ciencias de la Educación, establecido por el decreto 1569 de 1934, incluía en la sección pedagógica la asignatura Historia de la Educación en los tres primeros años de la carrera, con una intensidad horaria semanal de 3 horas cada una. El Plan de Estudios para las Escuelas Nomales Regulares establecido por el Decreto 2979 de 1945, incluía la asignatura Historia de la Educación, ubicada en el sexto y último año, con una intensidad horaria semanal de 2 horas. ${ }^{27}$

Aparte de esta referencia sobre la presencia de esas asignaturas, desconocemos los propósitos formativos, los contenidos enseñados y las perspectivas teóricas y metodológicas trabajadas en ellas; sin embargo, suponemos que su desarrollo correspondía al precario estado de la investigación histórico-educativa y a las concepciones historiográficas predominantes en la época; además, la baja intensidad horaria no podía permitir mayor profundidad en el desarrollo de la temática. Como dato adicional podemos mencionar que la Facultad de Ciencias de la Educación de la Universidad Nacional, fundada en 1932, contaba con Agustín Nieto Caballero, Juan Lozano y Lozano y Gabriel Anzola Gómez como profesores de His toria de la Educación.

\footnotetext{
${ }^{25}$ Téllez, Magaldy. "El concepto de genealogía en Foucault y la posibilidad de un replanteamiento de la investigación histónica en el ámbito de la educación”, Revista de Pedagogía, № 42, Escuela de Educación, Universidad Central de Venezuela, Caracas, 1995, pp. 71-82.

${ }^{26} \mathrm{P}$ ara el caso español, ver Escolano, Agustín. "Las escuelas normales. Siglo y medio de perspectiva histórica”, Revista de Educación, $\mathrm{N}^{\circ}$ 269, Madrid, 1982, pp. 55-76. Para el caso colombiano puede consultarse la revista Educación y Cultura, N²0, Bogotá, Julio de 1990, Monográfico “Reforma a las Normales".

${ }^{27}$ Tomado de Sáenz, Javier, et. al., Mirar la infancia... Vol. 2, Op. cit., pp. 507-534. 
La información sobre períodos posteriores es extraordinariamente difusa, tanto en referencia a las Escuelas Normales como a las Facultades de Educación. En las sucesivas reformas establecidas a partir de los años cincuenta se mantiene la ambigüedad sobre esta asignatura, con escasa presencia en los planes de estudio y una consideración mínima sobre su importancia formativa.

Creemos que la preocupación por incorporar esta temática al currículo como aspecto fundamental de la formación de docentes se ha ido dando paralelamente con el desarrollo de la investigación histórico-educativa y con las reflexiones alrededor de la pedagogía y la identidad profesional del maestro, en los años ochenta. Esto se puede constatar en algunos documentos presentados en los eventos y debates, ya mencionados, sobre la cris is de las instituciones formadoras de educadores, donde se plantea la necesidad de brindar una formación teórica en la pedagogía, desde la cual el maestro pueda reflexionar sobre sus concepciones, su saber y su quehacer, su condición social y cultural, sobre la historia de su oficio, sobre la enseñanza, sobre el sujeto al cual enseña, sobre su propia formación como sujeto de una práctica específica, históricamente determinada. Sin embargo, lo concreto es que dependiendo de la concepción curricular predominante en cada Facultad, o aún en cada programa académico, al tema se le ha dado mayor o menor importancia en los planes de estudio, bien como materia específica, como materia opcional, o como contenidos his tóricos parciales subsumidos en asignaturas más genéricas.

Esta situación, que en nuestro país expresa el desconocimiento o la subestimación permanentes por la temática, se manifiesta como crisis en otros contextos donde los estudios histórico-educativos han tenido una presencia significativa en los programas de formación de docentes.

El español Agustín Escolano ${ }^{28}$ se pregunta si la subestimación actual por la materia obedece a la debatida crisis general de la disciplina histórica o si es consecuencia de la imposición de políticas de formación de orientación fuertemente tecnicista y eficientis ta, o producto de ambas. Sin embargo, destaca a renglón seguido que el declinar de la Historia de la Educación como disciplina formativa coincide paradójicamente con la expansión sin precedentes de la investigación histórico-pedagógica. Agregando, además, que existe un interés renovado por esta historia sectorial en otros contextos historiográficos, principalmente en la historia social y cultural, cuyos objetos de estudio (mentalidades y comportamientos) precisan de las contribuciones históricopedagógicas para lograr una adecuada y completa explicación.

Estos interrogantes son importantes para analizar lo que ocurre actualmente en nuestra realidad educativa, donde nos vemos abocados a una nueva reforma de los programas de formación inicial y permanente de docentes. Existe un desarrollo creciente del campo de investigación en his toria de la educación y la pedagogía, y

\footnotetext{
${ }^{28}$ Escolano, Agustín.: “La investigación histórico-educativa y la formación de profesores”, Revista de Educación, № 157 , Madrid, enero-marzo 1994, p. 57. 
se abren posibilidades para incluirla en los currículos académicos como disciplina formativa. Aunque aún no es un tema central en las discusiones, se presentan nuevas condiciones para que esta temática pueda convertirse en una herramienta teórica y metodológica fundamental en los procesos formativos en todos los programas de educación. La normatividad vigente exige que esta dimensión sea parte constitutiva de los programas, que aparezca de manera explícita en el plan de estudios y con una definida intencionalidad formativa. Según esto, ya no se trata de consentir su inclusión en los nuevos proyectos curriculares por el simple interés particular de algunos docentes alrededor de esta temática, sino que en la actualidad se ha convertido en una exigencia académica ineludible. Al respecto, señalaré algunos elementos que confirman es ta exigencia. ${ }^{29}$

En el documento referenciado se plantea que los programas académicos que buscan formar profesionales de la educación deben fundamentarse en un enfoque claro de la profesión educativa, en la cual la pedagogía se constituye en la disciplina fundante de la formación y otorgándole al programa su identidad y su especificidad. A partir de este criterio general se establece que los programas se deben organizar en tomo a los siguientes núcleos básicos y comunes del saber pedagógico:

a) La educabilidad del ser humano.

b) La enseñabilidad de las disciplinas y saberes producidos por la humanidad, en el marco de sus dimensiones histórica, epistemológica, social y cultural y su transformación en contenidos y estrategias formativas, en virtud del contexto cognitivo, valorativo y social del aprendiz.

Se espera que este núcleo se haga presente en el currículo incorporando explícitamente el estudio de enfoques y teorías histórico-epistemológicas y de contenidos de las áreas o disciplinas del conocimiento objeto de enseñanza. La filosofía y la historia de las ciencias (naturales y sociales) y de la educación y la pedagogía constituyen campos de conocimientos que amplían el horizonte de acciones posibles del educador y le permiten adaptarse a las diversas circunstancias de la formación y responder creativamente a los problemas propios de su tarea social. La comprensión de las dimensiones histórica, epistemológica y social del conocimiento multiplica las posibilidades de tematizarlo de modo significativo para los estudiantes.

c) La estructura histórica y epistemológica de la pedagogía y sus posibilidades de interdisciplinariedad y de construcción y validación de teorías y modelos, así como las consecuencias formativas de la relación pedagógica.

\footnotetext{
${ }^{29}$ Cfr. Consejo Nacional de Acreditación. Criterios y procedimientos para la acreditación previa ..., op. cit., pp. $20-42$. 
La capacidad de elegir un enfoque pedagógico y de reconocer y desarrollar competencias en los alumnos depende de la comprensión que se tenga, más allá de los conocimientos y orientaciones particulares, de la estructura histórica y epistemológica del saber pedagógico. Los saberes específicos se comunican a través de las prácticas pedagógicas. El territorio del saber que corresponde a las prácticas pedagógicas es la pedagogía; es decir, el conocimiento que hace posible el tránsito del saber, de los espacios en donde se produce y se aplica, a los distintos espacios en los cuales se da la relación pedagógica. Esto implica un saber adicional a los saberes específicos y tan importante como ellos: el saber enseñar lo que se sabe. Por ello, la formación de los docentes debe implicar la historia de las prácticas educativas y el pensamiento de los grandes maestros que han contribuído a la definición de ese campo de debate que es la pedagogía.

d) Las realidades y tendencias sociales y educativas institucionales, nacionales e internacionales; y las dimensiones ética, cultural y política de la profesión educativa.

Se espera que este núcleo se haga presente en el currículo incorporando explícitamente temas de la historia universal y nacional de la pedagogía, en especial de aquella más relacionada con la especificidad del programa. Entre otros aspectos de la realidad educativa, debe incorporar la historia de la profesión, sus condiciones de realización, los compromisos sociales, éticos y políticos que de ella se derivan y las normas que la regulan.

Estas determinaciones recogen y refuerzan planteamientos hechos por diversos autores en el mismo sentido. Olga Lucía Zuluaga, al referirse a las pers pectivas de la investigación en el campo de la pedagogía, señala que es imprescindible convertir las Facultades de Educación en instituciones del saber pedagógico y en el lugar ideal para el trabajo histórico que demanda la recuperación de la práctica pedagógica. Esta recuperación debe apoyarse ante todo en trabajos his tóricos que permitan, por una parte, establecer el estatuto epistemológico de la Pedagogía y, por otra, las condiciones en que se le apropia como saber. Enfatiza, además, que la investigación acerca del proceso histórico de formación de la pedagogía como disciplina, es la base más firme para abordar las exigencias y perspectivas que le están planteando hoy, las teorías sobre el aprendizaje y el conocimiento, y la historia de las ciencias. Esta tarea debe as umirse des de la interioridad propia de la pedagogía; es decir, desde el reconocimiento de la existencia de un dominio discursivo propio que le permite redefinir, especificar y reordenar conceptos, modelos, justificaciones, aplicaciones e interrogantes que provienen de otros campos del conocimiento o que se elaboran en el interior de su saber, para evitar de esta manera, que volvamos a caer en el círculo vicioso de reducir la pedagogía a los procedimientos, tal como ha sido la costumbre en los programas de formación yen el ejercicio profesional de la docencia. ${ }^{30}$

\footnotetext{
${ }^{30}$ Zuluaga, Olga Lucía, Pedagogía e Historia, Op. cit., pp. 31-33. 
En un texto posterior se hacen algunas precisiones que resultan importantes para comprender esta perspectiva: "La historia del saber vivifica las comunidades disciplinarias, es parte de las certezas que deben cohesionarlas ... Así como las ciencias tienen su pasado actual, las dis ciplinas también lo pueden tener, pero con una diferencia, mientras las ciencias actualizan su pasado mediante la historia recurrente, las disciplinas en cambio lo deben hacer mediante las problematizaciones cuya solución se requiere para continuar su búsqueda, su construcción de sistematicidad". Además, al plantear que la historia de la pedagogía no es una labor propiamente de historiador, ni es tampoco un trabajo externo a la pedagogía misma, se quiere destacar que, en esta perspectiva, la reflexión his tórica hace parte de las labores teóricas internas de la pedagogía. Sin embargo, se reconoce el vínculo con la ciencia histórica en tanto las investigaciones se llevan a cabo mediante métodos correspondientes a este campo. $^{31}$

De igual manera, José Iván Bedoya hace un planteamiento interesante acerca de la historicidad de la pedagogía, del cual voy a presentar algunos aspectos. Señala que el propósito de este tipo de trabajo consiste en "analizar los diversos momentos que deteminan el saber pedagógico en su proceso de constitución teórica, apoyando tal análisis en los conceptos operacionales de la historia epistemológica". El punto de partida con respecto a la posibilidad y la necesidad de la fundamentación de la pedagogía es "entenderla como un saber o disciplina en construcción y en permanente reelaboración", por tanto, "más que entender el saber pedagógico como ya teminado, del que sólo se pretende su apropiación memorística, se debe trabajar en forma histórico-epistemológica, es decir, elaborando -o descubriendo, lo que supone necesariamente el enfoque investigativo- el proceso específico de su construcción o constitución histórica, tratando sobre todo de reconstruir teóricamente el problema que en su momento histórico fue planteado y cómo se le dio una respuesta determinada. Este es el sentido formativo de la historia de la pedagogía". Agrega, además, que "este enfoque histórico del saber pedagógico permitirá lograr una apropiación crítica y re-creativa del mismo, porque al entender epistemológica e históricamente cómo se han construído los objetos teóricos al interior del discurso pedagógico, se estará en capacidad no solo de comprender y conceptualizar los problemas y las condiciones teóricas que los hicieron posibles, sino poder encontrar y formular nuevos objetos de conocimiento". "Una de las razones de la recurrencia a los pedagógos (o a un autor del pasado) radica en el sentido formativo, - sin buscar una utilidad inmediata - que tiene el pensar o el re-pensar los conceptos o teorías pedagógicos que fueron elaborados en un momento histórico determinado ... Lo más es encial sería que este contacto (aproximación o apropiación) con el pasado de la pedagogía, como disciplina en formación, pueda afianzar o sustentar cada vez la vigencia actual del saber pedagógico ... No se puede pretender captar los contenidos de una teoría pedagógica reduciéndolos a meros resultados - que habría que aplicar en una situación concreta de enseñanza -: és ta es la obsesión

\footnotetext{
${ }^{31}$ Zuluaga, Olga L. y Echeverry, Jesús A., “E1 florecimiento de las investigaciones pedagógicas”, en Pedagogía Discurso y Poder, Bogotá, Compodic, 1990, pp. 185-186. 
por la práctica metodológica que siempre ha tenido el educador... Por el contrario, hay que insistir en la rigurosidad del proceso de constitución de las teorías pedagógicas al que el inacabamiento le es esencial, como dice G. Canguilhem". 32

Por su parte, Escolano, ${ }^{33}$ señala cuatro razones sobre el sentido que la investigación histórico-educativa puede tener en la fomación de maestros: En primer lugar, debemos considerar que todos los hechos y conceptualizaciones con que se va a enfrentar el futuro docente son categorías histórico-culturales y no se pueden analizar reductivamente como datos sincrónicos, de carácter teórico o técnico, aportados por las ciencias humanas y/o las ciencias de la educación. Temas como la condición infantil, la estructura y contenidos del currículo, los métodos y recursos tecnológicos utilizados, los roles docentes, las formas institucionales de escolarización, los sistemas de exámen y disciplina, el carácter social de la educación, la identidad profesional del maestro, entre otros, no pueden ser abordados solamente des de las contribuciones que la investigación empírica proporciona, porque las cuestiones que se implican en estos temas son de naturaleza cultural y responden siempre a una genealogía que condiciona y conforma su estructura y comportamientos. Resulta, por tanto, imprescindible recurrir a la historia para explicar cómo se han configurado estas realidades y conceptos.

En segundo lugar, la historia se constituye en un soporte necesario para la construcción o legitimación discursiva de las Teorías Educativas, en la medida en que éstas sean concebidas como teorías factuales y culturales, y no como simples teorías formales derivadas de presupuestos apriorísticos.

En tercer lugar, la historia de la educación y la pedagogía constituye una disciplina propedéutica para la formación de la identidad profesional del maestro. Ella nutre sus tradiciones, su prestigio, los elementos que definen su imágen y hasta los ingredientes que ha de internalizar para asumir con sentido su rol profesional. En esta relación que los docentes en formación establecerían con el pasado podría fundamentarse la búsqueda de las raíces que han configurado la profesión a la cual van a acceder.

Finalmente, la historia de la educación y la pedagogía puede ser orientada hacia el desarrollo crítico de los docentes, mostrando, por una parte, cómo las relaciones escuela-sociedad son complejas y a veces conflictivas, y evidenciando, por otra, los avances y retrocesos que siempre se operan en los procesos de cambio educativo y de modemización pedagógica.

Por tanto, es evidente que mediante el uso comprensivo de la disciplina histórica los futuros docentes pueden desarrollar una visión histórico-cultural de los hechos a los que han de enfrentarse, desarrollar un pensamiento crítico sobre la realidad social y educativa del país, identificar, apropiar y desarrollar los

\footnotetext{
${ }^{32}$ Bedoya, José Iván, Pedagogía ¿Enseñar a pensar? Reflexión filosófica sobre el proceso de enseñar, Santafé de Bogotá, Ecoe, 1998, pp. 93-105.

${ }^{33}$ Escolano, Agustín, “La investigación histórico-educativa ...”, Op. cit., pp. 57-58. 
conocimientos propios de su saber y de su quehacer, y re-construir los rasgos definitorios de su propia identidad personal, social y profesional. Esto sólo es posible reconociendo la importancia de proporcionar una formación histórica general y específica a los docentes, en todos los programas académicos que se ofrezcan; es decir, teniendo una sólida convicción sobre la utilidad de la historia como conocimiento formativo, con un fuerte potencial transformador de la sociedad y de la educación. Es indudable que la inclusión del estudio de esta parcela del conocimiento por si sola no va a resolver los complejos problemas que aquejan a la formación de docentes, pero, debemos aceptar que la ausencia de una pers pectiva histórica puede ser, entre otras, parte de la debilidad formativa de los actuales programas. Porque además de las escisiones ya mencionadas, agregamos otra al desvincular la formación y el ejercicio profesional de los docentes de sus tradiciones históricoeducativas, negándole la posibilidad de reconocerse y asumirse como sujetos portadores de saberes históricamente constituidos.

En lo planteado hasta ahora, tanto en lo referido a la constitución del campo de investigación como en los criterios es bozados sobre la reforma en curso, creemos haber encontrado suficientes elementos para justificar la inclusión de la his toria de la educación y la pedagogía como disciplina fomativa, "no sólo en el ámbito de la docencia -esto es, como contenidos de un syllabus curricular-, sino también en el de la investigación, es decir, como actividad heurística integrada en las acciones formativas para legitimar y motivar los desarrollos teóricos y prácticos de los programas de formación de los profesores". ${ }^{34}$

Para terminar debo expresar que soy consciente de que en estas notas quedan muchos asuntos por tratar y que los abordados requieren ser profundizados; pero considero que cumplen con su propósito si facilitan el debate sobre el tema y motivan el desarrollo teórico y práctico de propuestas que contribuyan a mejorar los procesos de fomación inicial y permanente de docentes.

\footnotetext{
${ }^{34}$ Ibid., pp. 58-59. Escolano presenta además una propuesta en torno a tres contenidos que pueden ser trabajados en todos los programas: la infancia, el currículo y la historia material de la escuela. Sugiere incluir la investigación históricoeducativa como práctica genealógica que aproxime a los alumnos al análisis genético y procesual de los hechos y conceptos que se expliciten en su programa formativo; y una práctica desde planteamientos próximos a los modelos de investigación-acción.
} 